\title{
Investigation of Hepatoprotective Activity of Induced Pluripotent Stem Cells in the Mouse Model of Liver Injury
}

\author{
Chih-Hung Chiang, ${ }^{1,2}$ Ching-Chih Chang, ${ }^{3,4}$ Hui-Chun Huang, ${ }^{3,5}$ Yi-Jen Chen,, ${ }^{3,6}$ \\ Ping-Hsing Tsai, ${ }^{1}$ Shaw-Yeu Jeng, ${ }^{2,3}$ Shuen-Iu Hung, ${ }^{1}$ Jung-Hung Hsieh, ${ }^{2}$ \\ Hsu-Shan Huang, ${ }^{7}$ Shih-Hwa Chiou, ${ }^{1,8}$ Fa-Yauh Lee, ${ }^{3,5}$ and Shou-Dong Lee ${ }^{3,5}$
}

${ }^{1}$ Institute of Pharmacology, National Yang-Ming University, No. 155, Section 2, Linong Street, Taipei 11221, Taiwan

${ }^{2}$ Division of Urology, Department of Surgery, Taipei Veterans General Hospital and Su-Ao \& Yuan-Shan Branch, No. 201, Section 2, Shih-Pai Road, Taipei 11217, Taiwan

${ }^{3}$ School of Medicine, National Yang-Ming University, No. 155, Section 2, Linong Street, Taipei 11221, Taiwan

${ }^{4}$ Division of General Medicine, Taipei Veterans General Hospital, No. 201, Section 2, Shih-Pai Road, Taipei 11217, Taiwan

${ }^{5}$ Division of Gastroenterology, Taipei Veterans General Hospital, No. 201, Section 2, Shih-Pai Road, Taipei 11217, Taiwan

${ }^{6}$ Department of Obstetrics and Gynecology, Taipei Veterans General Hospital, No. 201, Section 2, Shih-Pai Road, Taipei 11217, Taiwan

${ }^{7}$ Graduate Institute of Pharmacy, National Defense Medical Center, No. 161, Section 6, Minquan E. Road, Taipei 11490, Taiwan

${ }^{8}$ Department of Medical Research \& Education, Taipei Veterans General Hospital, No. 201, Section 2, Shih-Pai Road, Taipei 11217, Taiwan

Correspondence should be addressed to Shih-Hwa Chiou, shchiou@vghtpe.gov.tw and Fa-Yauh Lee, fylee@vghtpe.gov.tw

Received 5 April 2011; Accepted 27 May 2011

Academic Editor: Ken-ichi Isobe

Copyright (C) 2011 Chih-Hung Chiang et al. This is an open access article distributed under the Creative Commons Attribution License, which permits unrestricted use, distribution, and reproduction in any medium, provided the original work is properly cited.

To date liver transplantation is the only effective treatment for end-stage liver diseases. Considering the potential of pluripotency and differentiation into tridermal lineages, induced pluripotent stem cells (iPSCs) may serve as an alternative of cell-based therapy. Herein, we investigated the effect of iPSC transplantation on thioacetamide- (TAA-) induced acute/fulminant hepatic failure (AHF) in mice. Firstly, we demonstrated that iPSCs had the capacity to differentiate into hepatocyte-like cells (iPSC-Heps) that expressed various hepatic markers, including albumin, $\alpha$-fetoprotein, and hepatocyte nuclear factor- $3 \beta$, and exhibited biological functions. Intravenous transplantation of iPSCs effectively reduced the hepatic necrotic area, improved liver functions and motor activity, and rescued TAA-treated mice from lethal AHF. 1, $1^{\prime}$-dioctadecyl-3,3,3', $3^{\prime}$-tetramethylindocarbocyanine perchlorate cell labeling revealed that iPSCs potentially mobilized to the damaged liver area. Taken together, iPSCs can effectively rescue experimental AHF and represent a potentially favorable cell source of cell-based therapy.

\section{Introduction}

Acute or fulminant hepatic failure (AHF) is a severe liver injury accompanied by hepatic encephalopathy which causes multiorgan failure with a high mortality rate. The use of chemical reagents, such as thioacetamide (TAA) [1-4], acetaminophen [5], or galactosamine [6,7], may reproduce a number of important clinical characteristics of AHF, such as hypoglycemia, encephalopathy, and increased blood levels of hepatic enzymes. Consequently, the experimental models of AHF induced by these chemicals were widely used for the investigation of the pathophyisology and therapeutic strategies of AHF.

Liver transplantation has been shown to be an effective treatment for this liver failure. However, the drawback of the procedure is the shortage of donor organs combined with needing the immunosuppressant treatment [8]. Therefore, transplantation of hepatocytes or hepatocyte-like cells may provide great promise because cellular therapy is the relative simple and less invasive procedure. Recently, the use of embryonic stem cells (ESCs) has attracted attention for cellular therapy because of their capability to proliferation 
indefinitely and their potential to differentiate into all types of cells including hepatocytes [9-12]. Heo et al.'s study demonstrated that ESCs gave rise to functional hepatocytes that effectively integrated into and replaced injured parenchyma without formation of teratoma in the mouse model of liver injury [13]. There is evidence suggested that bone marrow $(\mathrm{BM})$ is another source of hepatic progenitors $[14,15]$. However, BM cells also contribute functionally and significantly to liver fibrosis [16]. We should be vigilant for the possibility of organ fibrosis induced by BM cell-based therapy.

Induced pluripotent stem cells (iPSCs) are novel stem cell populations induced from mouse and human adult somatic cells through reprogramming by transduction of defined transcription factors $[17,18]$. Recent studies further suggested that iPSCs were indistinguishable from ESCs in morphology, proliferative abilities, surface antigens, gene expressions, epigenetic status of pluripotent cell-specific genes, and telomerase activity [19]. Previous studies have shown that both human and mouse iPSCs can efficiently differentiate into functional hepatocytes in vitro $[20,21]$, which may be helpful in studying liver development and regenerative medicine. In the present study, we first differentiated iPSCs into iPSC-hepatocyte-like cells (iPCS-Heps) using a stepwise protocol and monitored the expression of hepatic markers on iPCS-Heps. Next, we transplanted iPSCs into TAA-treated mice and found that iPSCs were incorporated into livers and significantly improved the hepatic functions, motor activity, and mortality rate of mice. This cellular therapy opened an era for cell-based transplantation by overcoming the ethical controversy over ESCs.

\section{Materials and Methods}

2.1. In Vitro Hepatic Differentiation. The first clone of murine iPSCs Re7 were generated from mouse embryonic fibroblasts (MEF) derived from 13.5-day-old embryos of C57/B6 mice from our lab, and the secondary clone of murine iPSCs were kindly provided by Dr. Shinya Yamanaka. The iPSCs were cultured as previously described $[17,18]$. iPSCs were seeded at $2 \times 10^{4} \mathrm{cells} / \mathrm{cm}^{2}$ maintained in Dulbecco's modified Eagle's medium (DMEM; Invitrogen) containing $10 \%$ fetal bovine serum, $100 \mathrm{U} / \mathrm{mL}$ penicillin, and $100 \mu \mathrm{g} / \mathrm{mL}$ streptomycin in gelatin-coated plates, prior to induction by a 2 -step procedure. iPSCs were differentiated by using step-1 differentiating medium, consisting of DMED supplemented with $20 \mathrm{ng} / \mathrm{mL}$ HGF (Peprotech), $10 \mathrm{ng} / \mathrm{mL}$ bFGF (Peprotech), and $0.61 \mathrm{~g} / \mathrm{L}$ nicotinamide. After 7 days, step-1 differentiating medium was changed to step-2 maturation medium containing DMEM supplemented with $0.1 \mu \mathrm{M}$ Nicotinamide, dexamethasone (Dex; Sigma), and 1\% insulin-transferrin-selenium (ITS; Sigma). Medium changes were performed twice weekly. iPSCs treated with medium supplemented with no growth factors were used as the negative control. For embryoid body (EB) formation, iPSCs were dissociated into a single cell suspension by $0.25 \%$ trypsin-EDTA and plated onto nonadherent culture dishes in
DMEM with 15\% FBS, $100 \mathrm{mM}$ MEM nonessential amino acids, $0.55 \mathrm{mM}$ 2-mercaptoethanol, and antibiotics at a density of $2 \times 10^{6}$ cells $/ 100 \mathrm{~mm}$ plate. After 4 days in floating culture, EBs were transferred onto gelatin-coated plates and maintained in the same medium for 24 hours. EBs were then assigned for in vitro hepatocyte differentiation by using a two-step procedure as previously described, with some modifications [22].

2.2. RT-PCR. Total RNA was isolated from iPSCs and differentiating iPSCs using TRIzol Reagent (Invitrogen). The messenger RNA of hepatic specific markers including hepatocyte nuclear factor- $3 \beta$ (HNF-3 $\beta$ ), $\alpha$-fetoprotein (AFP), albumin (ALB), Transthyretin (TTR), $\alpha$-antitrypsin (AAT), and tyrosine-aminotransferase (TAT) was reverse transcribed to complementary DNA (cDNA) using a reverse transcription system (Promege). cDNA was amplified using Taq polymerase (Takara, Japan) at $94^{\circ} \mathrm{C}$ for 5 minutes, 35 cycles of $94^{\circ} \mathrm{C}$ for 30 seconds, $55^{\circ} \mathrm{C}$ for 30 seconds, $72^{\circ} \mathrm{C}$ for 30 seconds, and extension at $72^{\circ} \mathrm{C}$ for 10 minutes. The primers used are shown in Table 1. The relative expression of each gene was normalized against glyceraldehyde-3phosphate dehydrogenase (GAPDH).

2.3. Periodic Acid-Schiff (PAS) Stain for Glycogen. Cells were fixed in $4 \%$ paraformaldehyde and then permeabilized with $0.1 \%$ Triton X-100 for 10 minutes. Samples were then oxidized in $1 \%$ periodic acid for 5 minutes, rinsed 3 times in deionized $(\mathrm{d}) \mathrm{H}_{2} \mathrm{O}$, treated with Schiff's reagent for 15 minutes, and rinsed in $\mathrm{dH}_{2} \mathrm{O}$ for 5 to 10 minutes. Samples were counterstained with Mayer's hematoxylin for 1 minute and then rinsed in $(d) \mathrm{H}_{2} \mathrm{O}$. With 12 repetitive experiments, the samples were observed under light microscope.

2.4. Cellular Uptake Assay of Low-Density Lipoprotein $(L D L)$. The uptake capability of $1,1^{\prime}$-dioctadecyl-3,3,3',3'tetramethylindocarbocyanine perchlorate conjugated to acetylated-LDL (DiI-Ac-LDL; AbD Serotec) of iPSCs and differentiated cells was determined by fluorescent microscopy in 12 repetitive experiments. Cells were incubated with $20 \mu \mathrm{g} / \mathrm{mL}$ DiI-AC-LDL at $37^{\circ} \mathrm{C}$ for 24 hours. Incorporation of DiI-Ac-LDL into cells was visualized by fluorescence microscopy.

2.5. Immunostaining Analysis. Cells were fixed in 4\% paraformaldehyde and then permeabilized in PBS containing $1 \%$ bovine serum albumin and $0.3 \%$ Triton X-100 before incubating with primary antibodies in PBS/BSA at $4^{\circ} \mathrm{C}$ overnight. After washing with PBS, cells were incubated with rhodamine-conjugated secondary antibodies of anti-HNF$3 \beta$, anti-AFP, and antialbumin at room temperature for 30 minutes. After washing with PBS, the samples were mounting in Prolong Gold with 4,6-diamidino-2-phenylindole (DAPI; Molecular Probes). Fluorescence-labeled cells were observed under a fluorescent microscope.

2.6. Cell-Labeling Protocol. In this study, we transplanted DiI-labeled iPSC to trace the distribution of iPSC. In brief, 
TABLE 1: The sequences for the primers of RT-PCR.

\begin{tabular}{llc}
\hline Gene name & Primer sequence & Product length \\
\hline HNF-3 $3 \beta$ & Forward: CAGCTACACACACGCCAAAC & $204 \mathrm{bp}$ \\
& Backward: GGCACCTTGAGAAAGCAGTC & $173 \mathrm{bp}$ \\
AFP & Forward: TCGTATTCCAACAGGAGG & \\
& Backward: AGGCTTTTGCTTCACCAG & $260 \mathrm{bp}$ \\
ALB & Forward: GCTACGGCACAGTGCTTG & \\
& Backward: CAGGATTGCAGACAGATAGTC & $225 \mathrm{bp}$ \\
TTR & Forward: CTCACCACAGATGAGAAG & $484 \mathrm{bp}$ \\
AAT & Backward: GGCTGAGTCTCTCAATTC & $206 \mathrm{bp}$ \\
TAT & Forward: AATGGAAGAAGCCATTCGAT \\
& Backward: AAGACTGTAGCTGCTGCAGC & $155 \mathrm{bp}$ \\
\hline
\end{tabular}

Abbreviations: hepatocyte nuclear factor-3 $\beta$ : HNF-3 $\beta$; $\alpha$-fetoprotein: AFP; albumin: ALB; Transthyretin: TTR; $\alpha$-antitrypsin: AAT; tyrosine-aminotransferase: TAT; glyceraldehyde-3-phosphate dehydrogenase: GAPDH.

$1 \times 10^{6}$ mouse iPSCs were suspended in phosphate-buffered saline in the presence of DiI at a final concentration of $1 \mu \mathrm{g} / \mathrm{mL}$ and incubated for $10 \mathrm{~min}$ at $37^{\circ} \mathrm{C}$ followed by $5 \mathrm{~min}$ at $4^{\circ} \mathrm{C}$ and finally washed thrice with PBS.

2.7. Animal Model of Liver Injury. Male BALB/c mice, 7-8 weeks old with weighing 25-30 g, were used for our experiments. Fulminant hepatic failure was induced by intraperitoneal injection of thioacetamide (TAA) $(150 \mathrm{mg} / \mathrm{kg}$, Sigma) $[23,24]$. Mice were randomly divided into 2 groups: group 1 (phosphate buffer saline (PBS); $n=36)$ and group $2\left(2 \times 10^{6}\right.$ iPSCs; $n=23$ ) via tail vein injection. To avoid hypoglycemia and electrolyte imbalance [25], subcutaneous injections of a solution containing $10 \%$ glucose water mixed with lactate ringer $(25 \mathrm{~mL} / \mathrm{kg})$ were performed every 12 hours after the injection of TAA. Motor activity measurements and blood sampling were performed 24 hours after the administration of TAA to observe the immediate hepatic damage. In order to observe the prolonged hepatic damage, measurement of motor activity and survival rate and collection of blood samples were performed 72 hours after the administration of TAA in iPSCs- and PBS-treated groups. All mice were caged at $24^{\circ} \mathrm{C}$ with a 12 -h light-dark cycle and allowed free access to water and food. This study was approved by Taipei Veterans General Hospital Animal Committee, and the principles of Laboratory Animal Care were followed.

2.8. Measurement of Motor Activity. Motor activities in an open field were determined by using the Opto-Varimex animal activity meter (Columbus Instruments Inc.) [26]. The Opto-Varimex activity sensors utilize high-intensity, modulated infrared light beams to detect animal motion. Animals were housed in transparent cages (17 inches $\times 17$ inches $\times 8$ inches) through which 30 infrared beams pass in the horizontal plane, 15 on each axis. This device differentiates nonambulatory movements (scratching, gnawing) from ambulation on the basis of consecutive interruption of the infrared monitoring beams. An additional row of infrared beams in the horizontal plane ( 15 on each axis) about $10 \mathrm{~cm}$ above the floor was used to count the vertical movements. During the activity measurements, animals have no access to food or chow. All studies were performed under strictly standardized conditions in the dark room for 30 minutes. The counting numbers of the total movements, ambulatory movements, and vertical movements were separately recorded to reflect the motor activities of rats with fulminant hepatic failure. The motor activities were defined as zero in dead mice.

2.9. Liver Functional Tests. Biochemical parameters were measured using standard clinical methods. After anesthesia by ketamine $(10 \mathrm{mg} / 100 \mathrm{~g})$, intracardiac aspiration of blood was performed. A $0.8-0.9 \mathrm{~mL}$ of blood sample was collected from the heart into a pyrogen-free syringe containing $\sim 75$ units of heparin sodium, then placed in an ice bath and transported immediately to the laboratory. Serum biochemistry tests, including alanine aminotransferase (ALT), aspartate aminotransferase (AST), and total bilirubin, were measured by Vitro DT chemistry system (Johnson \& Johnson).

2.10. Statistical Analysis. Results were expressed as mean \pm S.D. Statistical analyses were performed by using unpaired Student's $t$-tests, and the survival rate analyses using log-rank test. Results were considered statistically significant at $P<$ 0.05 .

\section{Results}

3.1. In Vitro Differentiation of iPSCs into iPSC-Heps. We introduced retroviruses containing mouse Oct $3 / 4$, Sox2, Klf4, and c-Myc into MEF. These iPS clones were positive for 

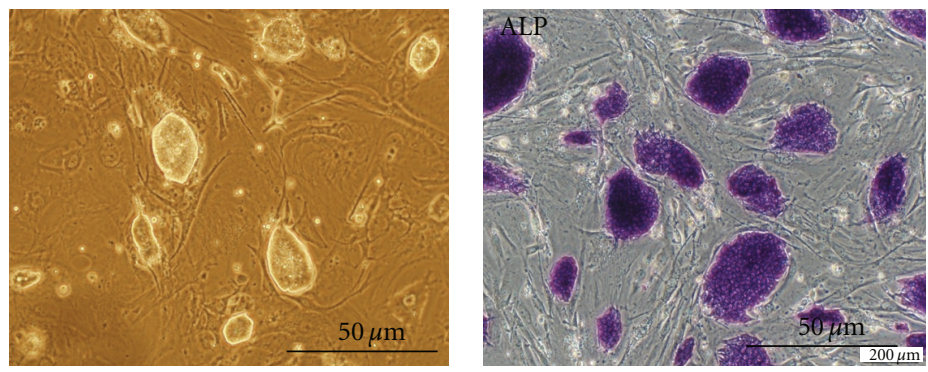

(a)
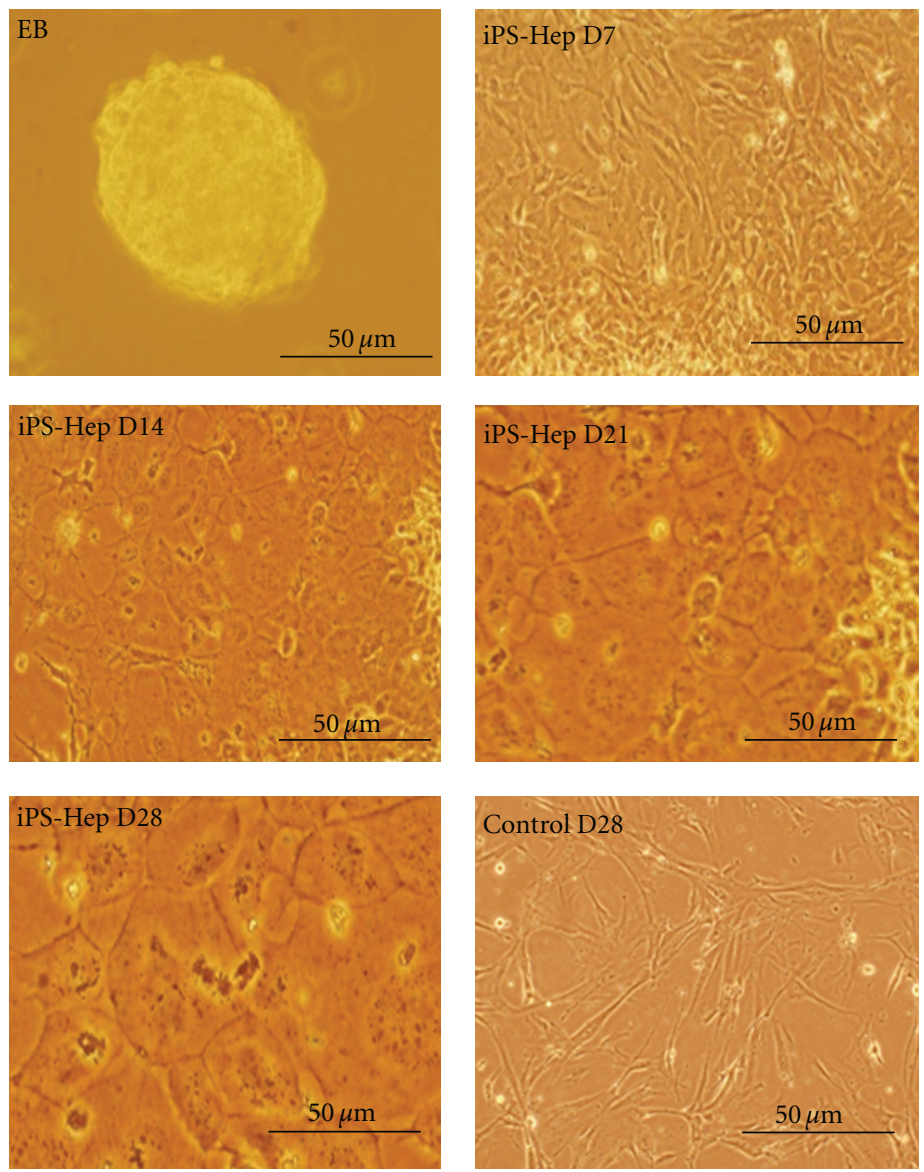

(b)

FIGURE 1: In vitro differentiation of iPSCs into iPSC-Heps. iPSCs were seeded at $2 \times 10^{4} \mathrm{cells} / \mathrm{cm}^{2}$, maintained in Dulbecco's modified Eagle's medium. (a) Left: Morphology of iPSC colonies. Right: iPSC colonies were positive for alkaline phosphate stain (purple). (b) The hepatogenic differentiation was induced by a 2-step procedure as described in Section 2. Morphology of undifferentiated and differentiated iPSCs was evaluated at different days after hepatogenic differentiation.

alkaline phosphatase (Figure 1(a)). Under hepatogenic condition, iPSC-derived embryoid bodies (EBs) (Figure 1(b), upper left) were shifted to hepatic differentiation media, and they gradually exhibited broadened and cuboidal morphology with increasing time of induction in vitro and eventually differentiated into iPSC-Heps at D7, D14, D21, and D28 (Figure 1(b)). Immunofluorescence staining with anti-ALB antibody and anti-AFP antibody was observed using the confocal microscope in iPSC-Heps, and showed increased expression of ALB and AFP in iPSC-Heps in the 28th day differentiation (Figure 2). In order to confirm the hepatic characteristics of iPSC-Heps RT-PCR and qRT-PCR analyses were performed to examine expression of hepatic-specific markers including HNF-3, AFP, ALB, TTR, AAT, and TAT (mean $\pm \mathrm{SD}, n=3$ ). Obviously, after week 2 post differentiation, the expression of hepatic-specific genes was further upregulated in iPSC-Heps compared to un-differentiated iPSCs (Figure 3(a)). Besides, expression of 

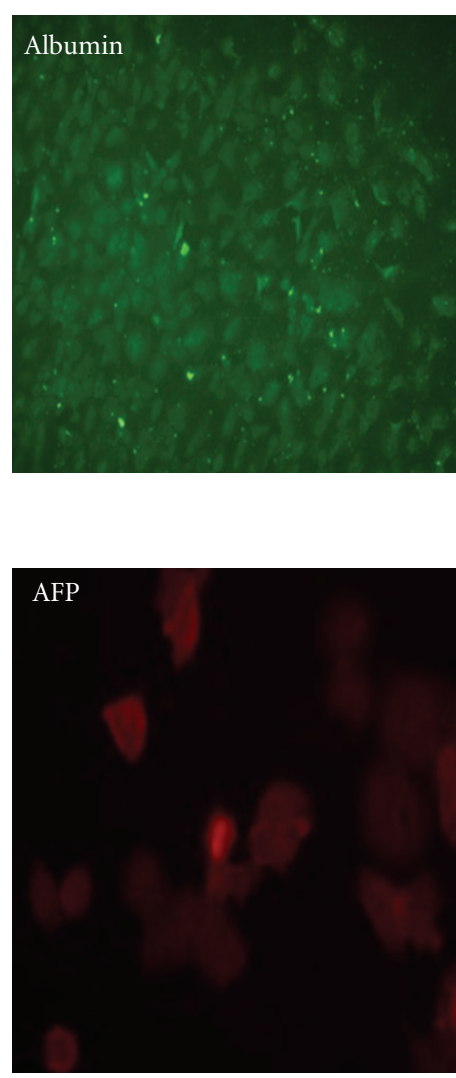

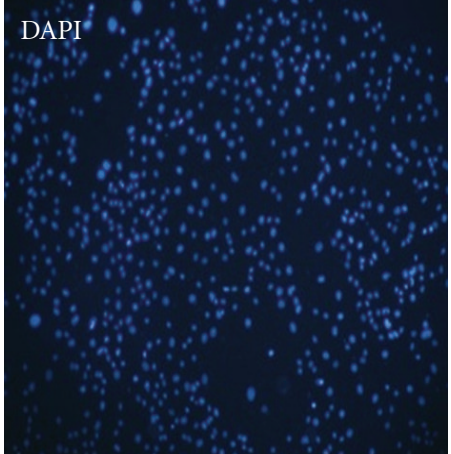

(a)
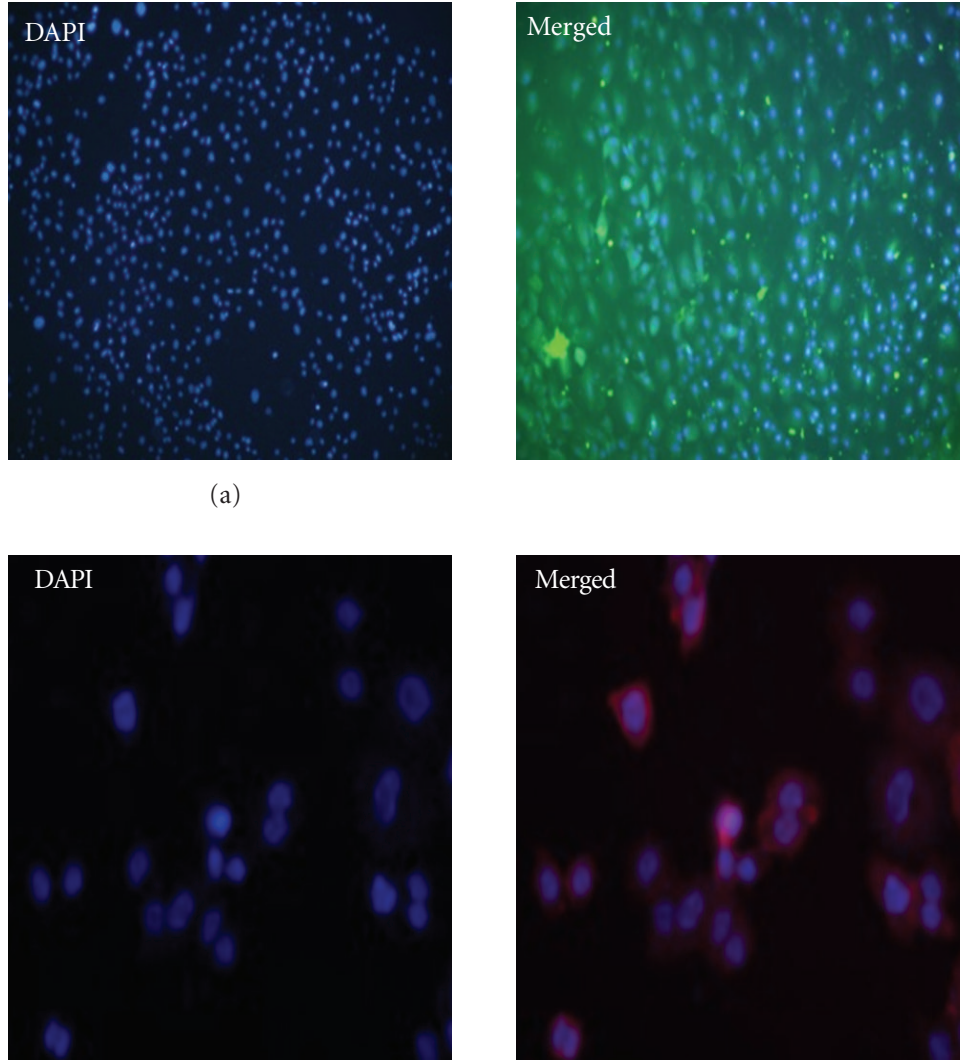

(b)

FIGURE 2: Immunofluorescence staining for several hepatocyte-specific markers in iPSC-Heps. Immunostaining imaging (800x) results showed that several hepatocyte-specific markers detected by using (a) anti-AFP antibody and (b) anti-albumin antibody in iPSC-Heps. Blue signal indicated DAPI-positive cells.

hepatic specific genes was significantly increased at week 2 after differentiation in HNF-3 $\beta$, TTR, AAT, and TAT, and at week 3 after differentiation in AFP and ALB, but not in undifferentiated iPSCs (Figure $3(\mathrm{~b}),{ }^{*} P<0.05$ versus iPSC).

3.2. Functional Characterization of iPSC-Heps. We further examined whether the iPSC-Heps with high expression of hepatic-specific genes also possessed biological hepatic functions. The uptake of LDL was measured by incorporation rate of DiI-Ac-LDL and the presence of stored glycogen was determined by PAS staining. Neither cellular uptake of LDL (mean $\pm \mathrm{SD}, n=12$ ) (Figure 4(a)) nor glycogen synthesis (mean $\pm \mathrm{SD}, n=12$ ) (Figure $4(\mathrm{~b})$ ) was observed in undifferentiated iPSCs, weeks 1 and 2 after iPSC-Heps differentiation. In contrast, iPSC-Heps showed significantly the ability to take up LDL (Figure 4(a)) and store glycogen (Figure 4(b)) over the three weeks of differentiation $(* P<$ 0.05 versus week 0$)$. Thus, the iPSC-Heps resemble hepatocytes both morphologically and functionally.

\subsection{Intravenous Transplantation of iPSCs Exhibited Beneficial} Effect on Survival Rate, Hepatic Encephalopathy, and Liver Functions in Mice with AHF. AHF is a severe liver disease accompanied by high mortality and hepatic encephalopathy that causes multiorgan failure. We assessed the therapeutic potential of iPSCs in TAA-induced AHF. We observed that in iPSCs-treated groups, $7 / 23(30.4 \%)$ mice were died 72 hours post administration of TAA, whereas 26/36 (72.2\%) mice were died in control groups. These results showed that the survival rate was significantly improved in mice receiving iPSC transplantation compared to controls (Figure 5(a), $P=$ 0.0018 by log-rank test). Furthermore, we examined whether iPSC transplantation could improve hepatic encephalopathy by assessing the motor activity of TAA-treated mice after iPSCs transplantation. Motor activity assay indicated that 72 hours after TAA administration, the total movements $(599 \pm$ 110 versus $246 \pm 71, P=0.006)$ and ambulatory movements $(364 \pm 68$ versus $155 \pm 45, P=0.009)$ were significantly increased in iPSCs-treated groups compared to PBS-treated groups (Figure 5(b)). These results demonstrated that iPSC treatment improved TAA-induced hepatic encephalopathy 72 hours after TAA injection.

To evaluate the effects of the 1st clone of iPSC Re7 from our lab on rescuing TAA-induced hepatic failure, we further evaluated whether this cell therapy also rescued liver functions in mice with AHF. There were robust increases in the levels of ALT, AST, and TBIL in PBS recipients 24 hours after TAA administration. These robust increases in hepatic biochemical parameters gradually decreased within 72 hours 

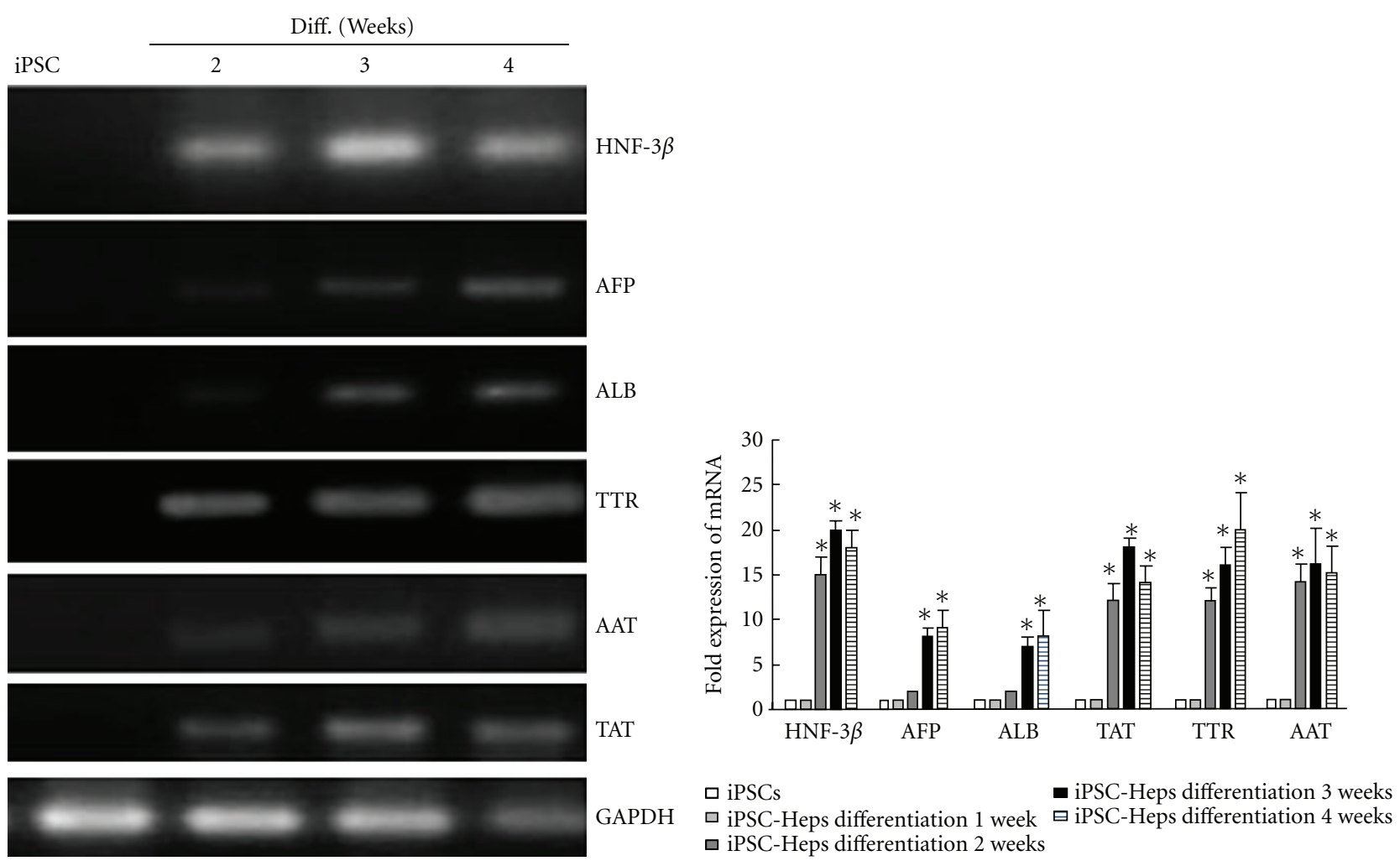

(a)

(b)

FIgURE 3: Gene expression of hepatic specific markers for iPSC-Heps. Expression of hepatic specific markers including HNF-3 $\beta$, AFP, ALB, TTR, AAT and TAT was detected by (a) RT-PCR and (b) q-RT-PCR during the hepatogenic differentiation course of iPSCs. Data shown here are the mean \pm SD. ${ }^{*} P<0.05$ versus iPSCs.

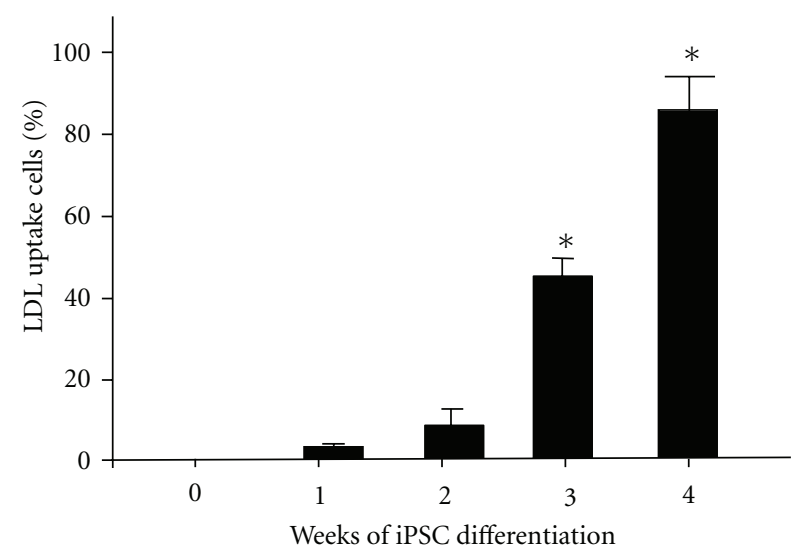

(a)

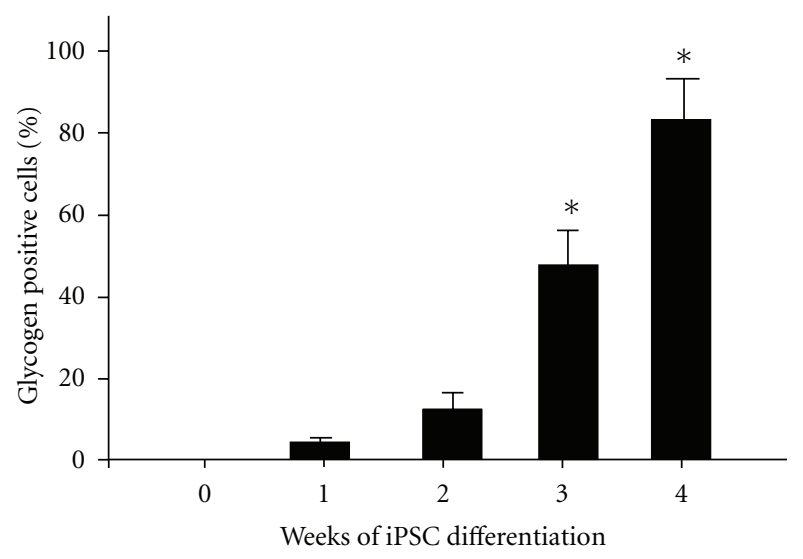

(b)

FIGURE 4: Functional characterization of iPSC-Heps. The uptake of LDL was measured by incorporation rate of DiI-Ac-LDL and the presence of stored glycogen was determined by PAS staining. Both (a) LDL uptake and (b) glycogen synthesis were evaluated over 4 weeks postdifferentiation. Data shown here are the mean \pm SD. ${ }^{*} P<0.05$ versus Week 0 .

(Table 2). The iPSC transplantation decreased all of these biochemical parameters at 24 hours and further significantly suppressed them at 72 hours after TAA administration (Table $2,{ }^{*} P<0.05$, iPSC versus PBS). Similar results were identified in the 2 nd clone of iPSC from Dr. Shinya Yamanaka (Table $3,{ }^{*} P<0.05$, iPSC versus PBS). These findings demonstrated that intravenously transplanted iPSCs can effectively rescue TAA-induced hepatic failure, restore liver functions, and improve survival in mice with AHF.

3.4. iPSC Transplantation Reduced Hepatic Necrotic Area in Mice with AHF. To explore whether transplantation of iPSCs 


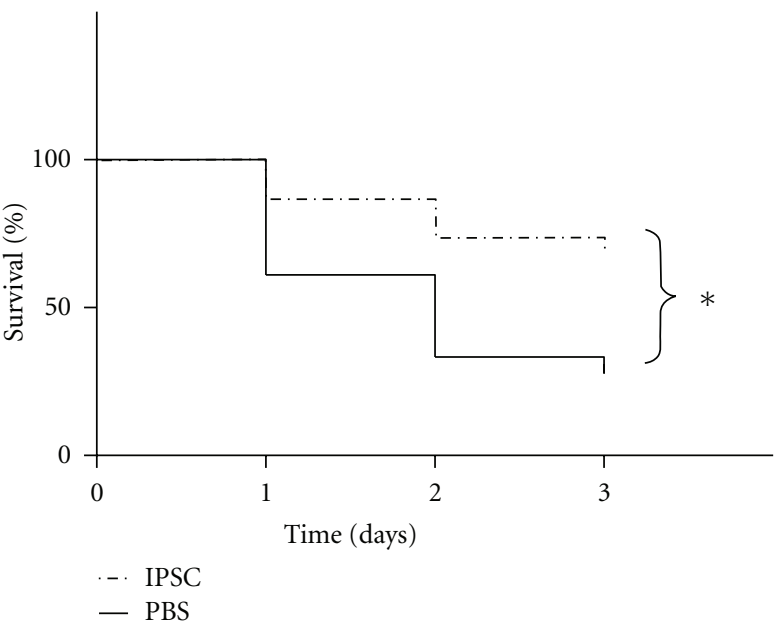

(a)

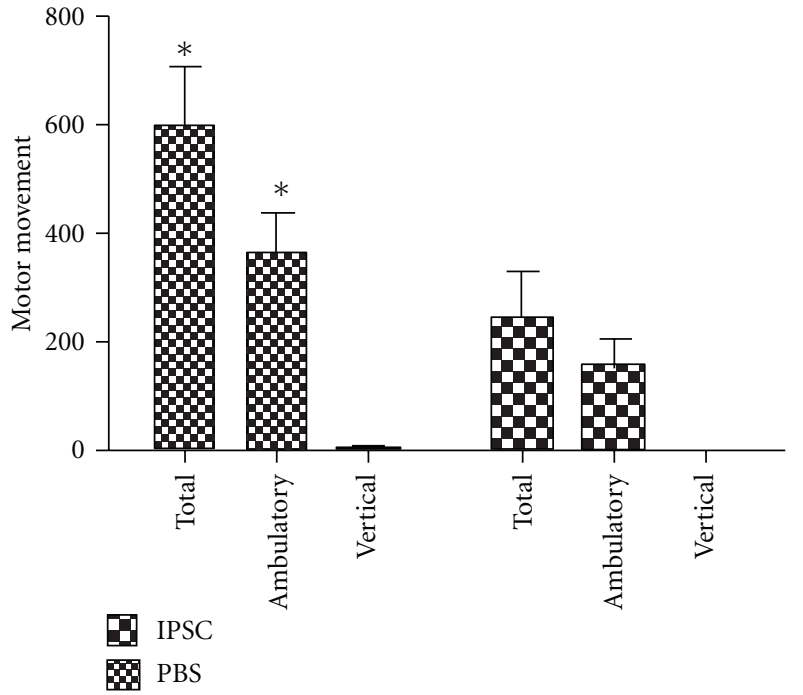

(b)

FIGURE 5: iPSCs increase the survival rate and motor activity of recipient mice. Male BALB/c mice were used for evaluation of the hepatoprotective activity of iPSC in the TAA-treated liver injury model. TAA $(150 \mathrm{mg} / \mathrm{kg})$ was given via intra-peritoneum injection and mice were received PBS or iPSCs $\left(2 \times 10^{6}\right.$ cells) via tail vein injection to determinate (a) the survival rate and (b) motor movements. Data shown here are the mean $\pm \mathrm{SD} .{ }^{*} P<0.05$ versus PBS.

TABLE 2: Effect of intravenous transplantation of PBS or iPSCs clone 1 Re7 from our lab on hepatic biochemical parameters in TAA-treated recipients.

\begin{tabular}{|c|c|c|c|}
\hline & Alanine aminotransferase (ALT; IU/L) & Aspartate aminotransferase (AST; IU/L) & Total bilirubin (TBIL; mg/dL) \\
\hline \multicolumn{4}{|c|}{ Recipient after 24 hours } \\
\hline PBS & $20702 \pm 1631$ & $6956 \pm 522$ & $2.0 \pm 0.3$ \\
\hline iPSCs & $5175 \pm 509^{*}$ & $1932 \pm 290^{*}$ & $1.3 \pm 0.2^{*}$ \\
\hline \multicolumn{4}{|c|}{ Recipient after 72 hours } \\
\hline PBS & $1721 \pm 497$ & $1116 \pm 286$ & $1.0 \pm 0.2$ \\
\hline iPSCs & $312 \pm 62^{*}$ & $248 \pm 76^{*}$ & $0.5 \pm 0.1^{*}$ \\
\hline
\end{tabular}

Results were expressed as mean \pm SD. ${ }^{*} P<0.05$ versus PBS.

repairs hepatic necrosis, we compared H\&E-stained liver sections from TAA-treated mice that received infusion of either iPSCs or PBS via tail vein. After TAA treatment, AHF happened immediately with obvious hepatic necrosis area. However, histological examination revealed that the rescuing effect of transplantation of iPSC clone 1 from our lab was initially observable in 24 hours than PBS group, and the numbers of necrotic areas (black arrow) in iPSC transplantation start to decrease significantly (Figures $6(\mathrm{a})$ and $6(\mathrm{~b}),{ }^{*} P<0.05$ versus PBS). Furthermore, lymphocyte infiltration (hallow arrow) and necrotic areas (black arrow) were remarkably diminished in 72 hours in iPSC transplantation (Figures 6(a) and 6(b), ${ }^{*} P<0.05$ versus PBS) in iPSC clone 1 from our lab. Similar results were identified at iPSC clone 2 from Dr. Shinya Yamanaka in 24 and 72 hours (Figure 6(c), ${ }^{*} P<0.05$ versus PBS).

3.5. iPSCs Mobilized to the Damage Area of TAA-Injured Liver. To further explore the fate of intravenously transplanted iPSCs from tail vein, we labeled iPSCs with DiI and explored if the iPSCs could migrate and incorporate into the damaged liver in TAA-treated mice. As detected by immunofluorescence staining, we observed that DiI-labeled iPSCs were found around central veins and were scattered the damaged liver areas (Figure 7(a) light field and 7(b) dark field). This implied that iPSCs can migrate from the peripheral space into areas of damaged liver in TAA-treated mice and may have favorable effects on improving liver functions and motor activity, and rescuing TAA-treated mice from lethal AHF. These hepatoprotective properties may subsequently improve hepatic encephalopathy and impaired motor activity.

\section{Discussion}

In this present study, we first showed that iPSC-Heps can be generated from iPSCs using the stepwise differentiation protocol. We demonstrated that intravenous transplantation of iPSCs can mobilize to the damaged liver area and extensively reduced the hepatic necrotic area, improved liver functions and motor activity, and rescued TAA-treated mice from lethal in mice with TAA-induced liver failure. Taken 

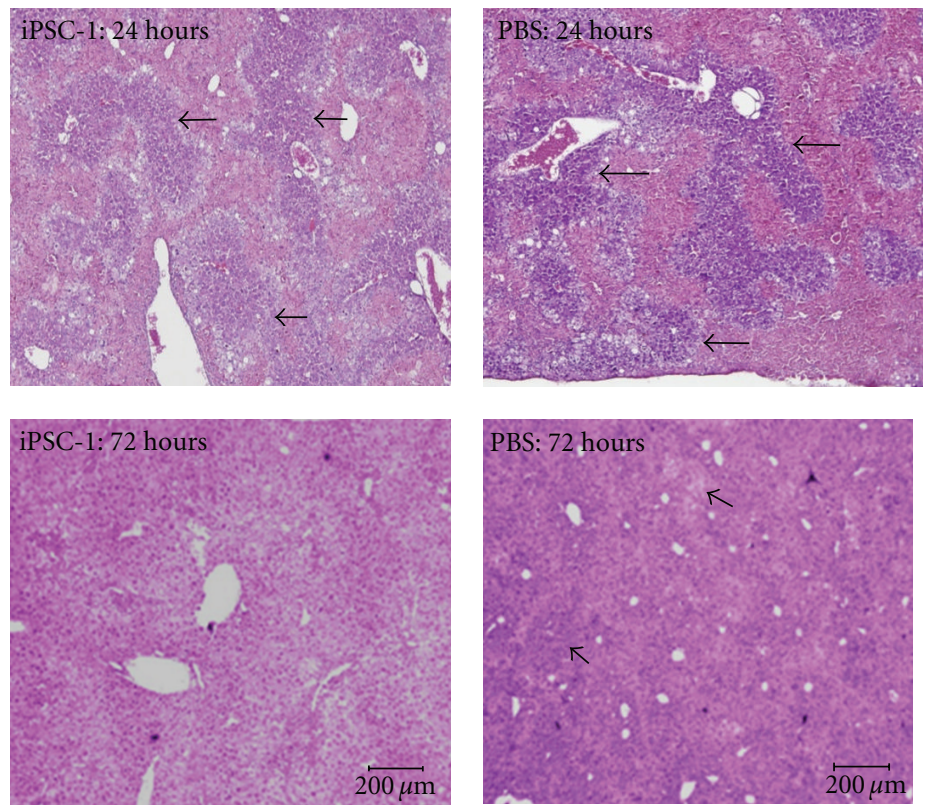

(a)
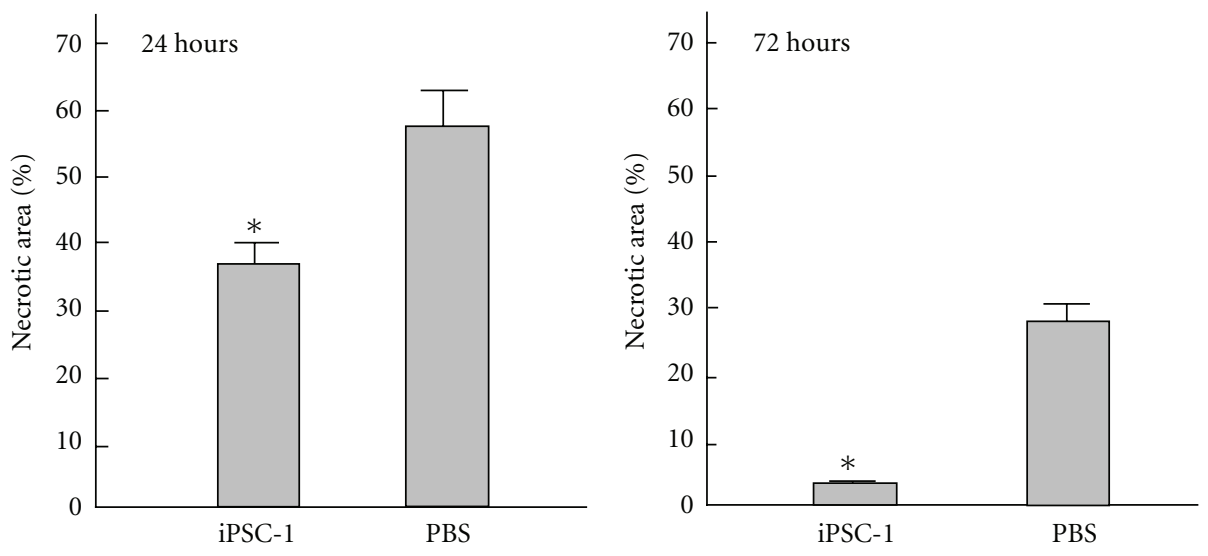

(b)
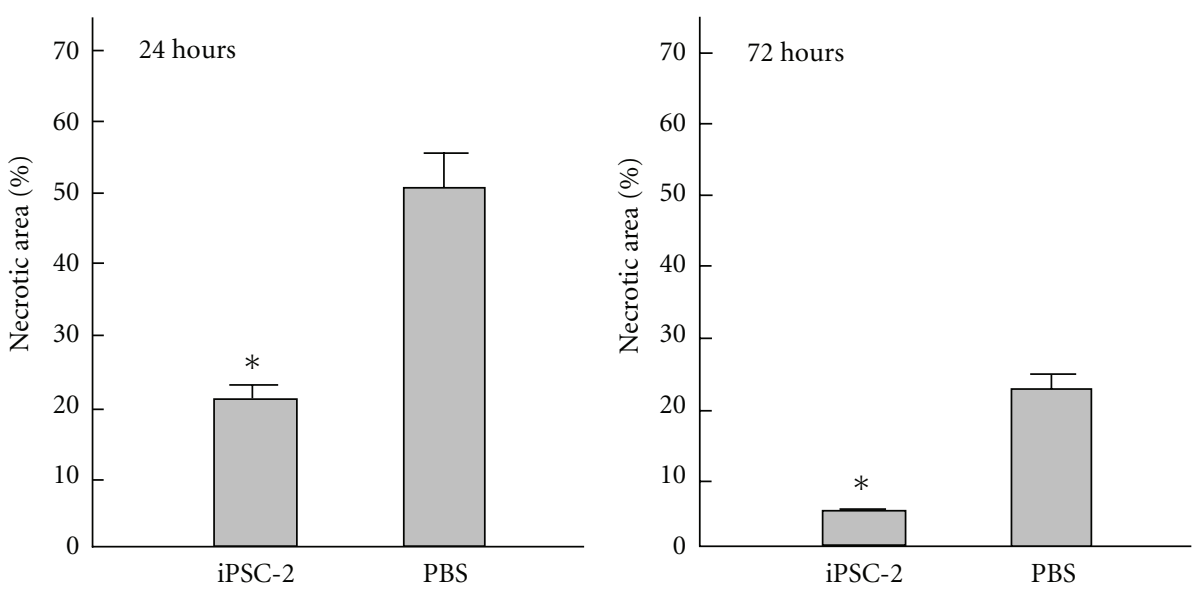

(c)

FIGURE 6: Effects of iPS cells on histopathological changes in recipient mice. Results showed the representative H\&E stain of TAA-treated liver tissue receiving iPSCs or PBS treatment after 24 hours or 72 hours in iPSC clone 1 from our lab and clone 2 from Shinya Yamanaka. In (a) and (b), the necrotic areas in iPSC-treated group were significantly reduced than PBS-treated group in 24 hours and 72 hours after TAA administration in iPSC clone 1 . In (c), similar results were identified in iPSC clone $2 .{ }^{*} P<0.05$ versus PBS. 


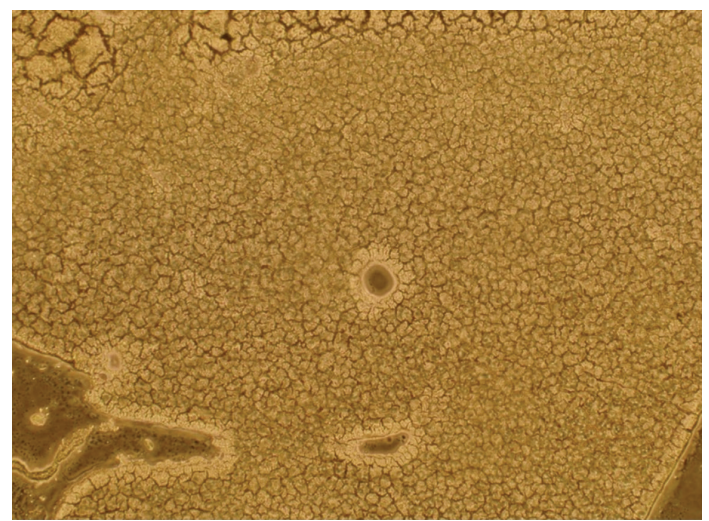

(a)

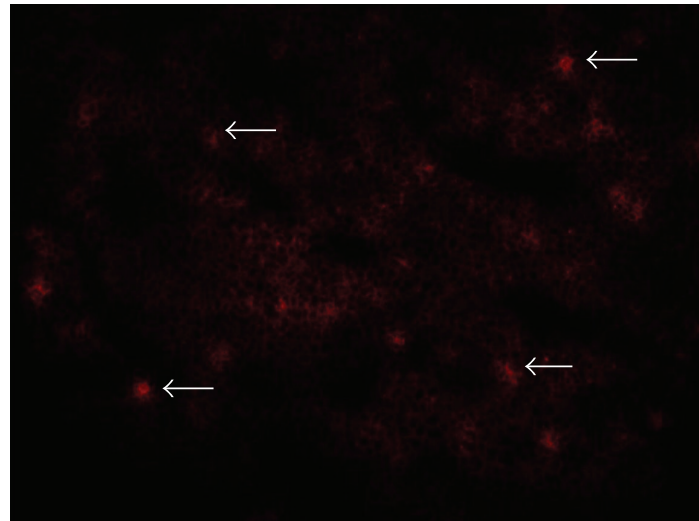

(b)

Figure 7: Mobilization of DiI-labeled iPSCs to the liver of mice with AHF. DiI fluorescent staining was used to trace the migration of the cells in the body. The transplanted DiI-labeled iPSCs was indicated as red spots under dark field microscopy (b) in the damaged liver area in TAA-treated mice. White arrows indicated the DiI-labeled iPSC and results indicated histological examination under (a) light field; (b) dark field.

TABLE 3: Effect of intravenous transplantation of PBS or iPSCs clone 2 from Dr. Shinya Yamanaka on hepatic biochemical parameters in TAA-treated recipients.

\begin{tabular}{|c|c|c|c|}
\hline & Alanine aminotransferase (ALT; IU/L) & Aspartate aminotransferase (AST; IU/L) & Total bilirubin (TBIL; mg/dL) \\
\hline \multicolumn{4}{|c|}{ Recipient after 24 hours } \\
\hline PBS & $17768.2 \pm 786.9$ & $6875.2 \pm 929.1$ & $3.87 \pm 0.45$ \\
\hline iPSCs & $3157.4 \pm 336.8^{*}$ & $1512.9 \pm 257.1^{*}$ & $1.53 \pm 0.46^{*}$ \\
\hline \multicolumn{4}{|c|}{ Recipient after 72 hours } \\
\hline PBS & $1553.4 \pm 67.1$ & $984.7 \pm 26.2$ & $1.53 \pm 0.18$ \\
\hline iPSCs & $217.7 \pm 7^{*}$ & $175.1 \pm 8.4^{*}$ & $0.92 \pm 0.13^{*}$ \\
\hline
\end{tabular}

Results were expressed as mean \pm SD. ${ }^{*} P<0.05$ versus PBS.

together, our data showed that iPSCs can effectively rescue experimental AHF and may provide an alternative cell source to hepatocyte transplantation for acute/fulminant liver diseases. Nevertheless, the underlying mechanisms of iPSCmediated therapeutic effect remained largely unknown.

It has been clearly demonstrated that mesenchymal stem cells (MSCs) have a capacity to mobilize and integrate into damaged tissues and provide immunomodulatory effects to undergo tissue repair via paracrine effects [27]. Consequently, MSC transplantation has been regarded as a new clinical approach for tissue regeneration. Intravenous transplantation of MSCs has been shown to mobilize to the damaged liver and improved hepatic functions in mice with AHF [28]. Interestingly, in the present study, the distribution of DiI-labeled iPSCs demonstrated that iPSCs could also mobilize to the damaged area of liver, in a manner similar to that of MSCs. BM-derived MSC is widely used for MSC-based regeneration study, whereas such cell is relatively inaccessible $[29,30]$, and the cell number and the differentiating potential of BM-derived MSCs were decreased with age [31]. Given that iPSCs were generated from somatic cells and exhibited remarkable therapeutic potential on fulminant hepatic failure, iPSC could be another choice for cellbased therapy against fulminant hepatic failure.
Cross-gender and whole-liver transplantation studies in rodents indicate that BM-derived or extrahepatic stem cells can differentiate into hepatocytes [32]. Previous studies also showed that early hepatic precursors can be generated from ESCs in vitro and these precursors can differentiate into functional hepatocytes that rescued and incorporated into diseased liver parenchyma [13]. Although iPSCs possess pluripotent properties similar to those of ESCs, whether the transplanted iPSCs differentiated into hepatocytes or iPSCHeps after incorporation into the liver area was unknown. We speculated that there were some possible mechanisms for iPSC-mediated hepatoprotective effect, including (a) restoration of impaired hepatic function by engrafted iPSCs that may undergo differentiation and have mature hepatocyte functions in vivo, (b) stimulating the proliferation of new functional hepatocytes that may restore hepatic function, via a paracrine manner, and (c) providing certain immunomodulatory effects similar to those of MSCs and repair damaged tissue. Nevertheless, further investigations will be required to clarify these speculations.

Previous studies have demonstrated that iPSCs can differentiate into different types of cells including cardiomyocytes, endothelial cells, and neural cells in vitro [33-36]. 
Given the potential to generate patient-specific cell populations without the need for human embryonic cells, iPSC technology has received great excitement by research and medical communities. However, many questions regarding the actual molecular process of induced reprogramming remain unanswered and need to be addressed. Teratoma formation [37] and genomic instability [38] have been reported to be the major risk in the transplantation of iPSCs generated by four exogenously introduced genes (Oct4/Sox $2 / \mathrm{Klf} 4 / \mathrm{c}-$ Myc). Notably, there are no observable tumor-like structures in the liver after a follow-up observation for one month after iPSC transplantation in the present study. To fully exclude the risk of teratoma formation and nonviral vector or replaced the pro-oncogens of c-Myc and Klf4 may be a more safe way and should be developed in the future study.

Comparing with MSCs and ESCs, iPSCs are relatively accessible and with less ethical issues and immunorejection. Our results provided evidence that iPSCs can migrate from systemic circulation to the damaged liver in TAA-treated mice and immediately improve liver functions and rescue survival benefit shortly after transplantation. Therefore, this iPSC transplantation may provide an alternative of therapeutic strategy for patients with acute/fulminant hepatic failure.

\section{Conflict of Interest}

The authors declare that they do not have anything to disclose regarding funding from industries or conflict of interest with respect to this manuscript.

\section{Acknowledgments}

The authors thank Dr. Shinya Yamanaka, the RIKEN BRC, Japan, for kindly providing iPSCs in this study. This study was supported by research Grants from NSC-(97-3111-B075-001-MY3), Taipei Veterans General Hospital (V97B1006, E1-008, ER2-018, ER3-005, F-001), the Joint Projects of UTVGH (96-P1-07), Yen-Tjing-Ling Medical Foundation, and National Yang-Ming University (Ministry of Education, Aim for the Top University Plan), Taiwan, and Taipei Veterans General Hospital, Yuan-Shan branch (YSVH-9902), Yi-Lan, Taiwan. C.-H. Chiang and C.-C. Chang contributed equally to this work.

\section{References}

[1] E. Chieli and G. Malvaldi, "Role of the microsomal fadcontaining monooxygenase in the liver toxicity of thioacetamide S-oxide," Toxicology, vol. 31, no. 1, pp. 41-52, 1984.

[2] V. Pallottini, C. Martini, A. M. Bassi, P. Romano, G. Nanni, and A. Trentalance, "Rat HMGCoA reductase activation in thioacetamide-induced liver injury is related to an increased reactive oxygen species content," Journal of Hepatology, vol. 44, no. 2, pp. 368-374, 2006.

[3] H. Shapiro, M. Ashkenazi, N. Weizman, M. Shahmurov, H. Aeed, and R. Bruck, "Curcumin ameliorates acute thioacetamide-induced hepatotoxicity," Journal of Gastroenterology and Hepatology, vol. 21, no. 2, pp. 358-366, 2006.
[4] B. K. Gunawan, Z. X. Liu, D. Han, N. Hanawa, W. A. Gaarde, and N. Kaplowitz, "c-Jun N-terminal kinase plays a major role in murine acetaminophen hepatotoxicity," Gastroenterology, vol. 131, no. 1, pp. 165-178, 2006.

[5] A. P. Margeli, L. Papadimitriou, S. Ninos, E. Manolis, M. G. Mykoniatis, and S. E. Theocharis, "Hepatic stimulator substance administration ameliorates liver regeneration in an animal model of fulminant hepatic failure and encephalopathy," Liver International, vol. 23, no. 3, pp. 171-178, 2003.

[6] B. L. Blitzer, J. G. Waggoner, and E. A. Jones, "A model of fulminant hepatic failure in the rabbit," Gastroenterology, vol. 74, no. 4, pp. 664-671, 1978.

[7] M. E. Horowitz, D. F. Schafer, and P. Molnar, "Increased blood-brain transfer in a rabbit model of acute liver failure," Gastroenterology, vol. 84, no. 5, pp. 1003-1011, 1983.

[8] N. Selzner, D. R. Grant, I. Shalev, and G. A. Levy, "The immunosuppressive pipeline: meeting unmet needs in liver transplantation," Liver Transplantation, vol. 16, no. 12, pp. 1359-1372, 2010.

[9] N. Lumelsky, O. Blondel, P. Laeng, I. Velasco, R. Ravin, and R. McKay, "Differentiation of embryonic stem cells to insulinsecreting structures similar to pancreatic islets," Science, vol. 292, no. 5520, pp. 1389-1394, 2001.

[10] E. A. Jones, D. Tosh, D. I. Wilson, S. Lindsay, and L. M. Forrester, "Hepatic differentiation of murine embryonic stem cells," Experimental Cell Research, vol. 272, no. 1, pp. 15-22, 2002.

[11] Y. Kumashiro, K. Asahina, R. Ozeki et al., "Enrichment of hepatocytes differentiated from mouse embryonic stem cells as a transplantable source," Transplantation, vol. 79, no. 5, pp. 550-557, 2005.

[12] H. Yamamoto, G. Quinn, A. Asari et al., "Differentiation of embryonic stem cells into hepatocytes: biological functions and therapeutic application," Hepatology, vol. 37, no. 5, pp. 983-993, 2003.

[13] J. Heo, V. M. Factor, T. Uren et al., "Hepatic precursors derived from murine embryonic stem cells contribute to regeneration of injured liver," Hepatology, vol. 44, no. 6, pp. 1478-1486, 2006.

[14] N. D. Theise, M. Nimmakayalu, R. Gardner et al., "Liver from bone marrow in humans," Hepatology, vol. 32, no. 1, pp. 11$16,2000$.

[15] M. R. Alison, R. Poulsom, R. Jeffery et al., "Hepatocytes from non-hepatic adult stem cells," Nature, vol. 406, no. 6793, p. 257, 2000.

[16] F. P. Russo, M. R. Alison, B. W. Bigger et al., "The bone marrow functionally contributes to liver fibrosis," Gastroenterology, vol. 130, no. 6, pp. 1807-1821, 2006.

[17] K. Takahashi and S. Yamanaka, "Induction of pluripotent stem cells from mouse embryonic and adult fibroblast cultures by defined factors," Cell, vol. 126, no. 4, pp. 663-676, 2006.

[18] K. Okita, T. Ichisaka, and S. Yamanaka, "Generation of germline-competent induced pluripotent stem cells," Nature, vol. 448, no. 7151, pp. 313-317, 2007.

[19] S. Yamanaka, "A fresh look at iPS cells," Cell, vol. 137, no. 1, pp. 13-17, 2009.

[20] K. Si-Tayeb, F. K. Noto, M. Nagaoka et al., "Highly efficient generation of human hepatocyte-like cells from induced pluripotent stem cells," Hepatology, vol. 51, no. 1, pp. 297-305, 2010.

[21] P. Sancho-Bru, P. Roelandt, N. Narain et al., "Directed differentiation of murine-induced pluripotent stem cells to 
functional hepatocyte-like cells," Journal of Hepatology, vol. 54, no. 1, pp. 98-107, 2011.

[22] Z. Song, J. Cai, Y. Liu et al., "Efficient generation of hepatocyte-like cells from human induced pluripotent stem cells," Cell Research, vol. 19, no. 11, pp. 1233-1242, 2009.

[23] T. C. Jeong, H. K. Gu, J. I. Park et al., "Pretreatment of male $\mathrm{BALB} / \mathrm{c}$ mice with $\beta$-ionone potentiates thioacetamideinduced hepatotoxicity," Toxicology Letters, vol. 105, no. 1, pp. 39-46, 1999.

[24] T. Hayase, Y. Yamamoto, K. Yamamoto, H. Abiru, Y. Nishitani, and Y. Fukui, "Relationship between cocaine-induced hepatotoxic neurobehavioral and biochemical changes in mice: the antidotal effects of buprenorphine," Life Sciences, vol. 67, no. 1, pp. 45-52, 2000.

[25] S. H. Gammal, A. S. Basile, D. Geller, P. Skolnick, and E. A. Jones, "Reversal of the behavioral and electrophysiological abnormalities of an animal model of hepatic encephalopathy by benzodiazepine receptor ligands," Hepatology, vol. 11, no. 3, pp. 371-378, 1990.

[26] J. Ribeiro, B. Nordlinger, F. Ballet et al., "Intrasplenic hepatocellular transplantation corrects hepatic encephalopathy in portacaval-shunted rats," Hepatology, vol. 15, no. 1, pp. 12-18, 1992.

[27] H. Yagi, A. Soto-Gutierrez, B. Parekkadan et al., "Mesenchymal stem cells: mechanisms of immunomodulation and homing," Cell Transplantation, vol. 19, no. 6-7, pp. 667-679, 2010.

[28] T. K. Kuo, S. P. Hung, C. H. Chuang et al., "Stem cell therapy for liver disease: parameters governing the success of using bone marrow mesenchymal stem cells," Gastroenterology, vol. 134, no. 7, pp. 2111-e3, 2008.

[29] H. K. Väänänen, "Mesenchymal stem cells," Annals of Medicine, vol. 37, no. 7, pp. 469-479, 2005.

[30] R. Cancedda, G. Bianchi, A. Derubeis, and R. Quarto, "Cell therapy for bone disease: a review of current status," Stem Cells, vol. 21, no. 5, pp. 610-619, 2003.

[31] S. V. Tokalov, S. Grüner, S. Schindler, G. Wolf, M. Baumann, and N. Abolmaali, "Age-related changes in the frequency of mesenchymal stem cells in the bone marrow of rats," Stem Cells and Development, vol. 16, no. 3, pp. 439-446, 2007.

[32] M. R. Alison, R. Poulsom, R. Jeffery et al., "Hepatocytes from non-hepatic adult stem cells," Nature, vol. 406, no. 6793, p. 257, 2000.

[33] A. Moretti, M. Bellin, A. Welling et al., "Patient-specific induced pluripotent stem-cell models for long-QT syndrome," The New England Journal of Medicine, vol. 363, no. 15, pp. 1397-1409, 2010.

[34] D. Taura, M. Sone, K. Homma et al., "Induction and isolation of vascular cells from human induced pluripotent stem cells-brief report," Arteriosclerosis, Thrombosis, and Vascular Biology, vol. 29, no. 7, pp. 1100-1103, 2009.

[35] S. Karumbayaram, B. G. Novitch, M. Patterson et al., "Directed differentiation of human-induced pluripotent stem cells generates active motor neurons," Stem Cells, vol. 27, no. 4, pp. 806-811, 2009.

[36] S. J. Chen, C. M. Chang, S. K. Tsai et al., "Functional improvement of focal cerebral ischemia injury by subdural transplantation of induced pluripotent stem cells with fibrin glue," Stem Cells and Development, vol. 19, no. 11, pp. 1757$1767,2010$.
[37] K. Miura, Y. Okada, T. Aoi et al., "Variation in the safety of induced pluripotent stem cell lines," Nature Biotechnology, vol. 27, no. 8, pp. 743-745, 2009.

[38] C. E. Pasi, A. Dereli-Öz, S. Negrini et al., "Genomic instability in induced stem cells," Cell Death and Differentiation, vol. 18, no. 5, pp. 745-753, 2011. 

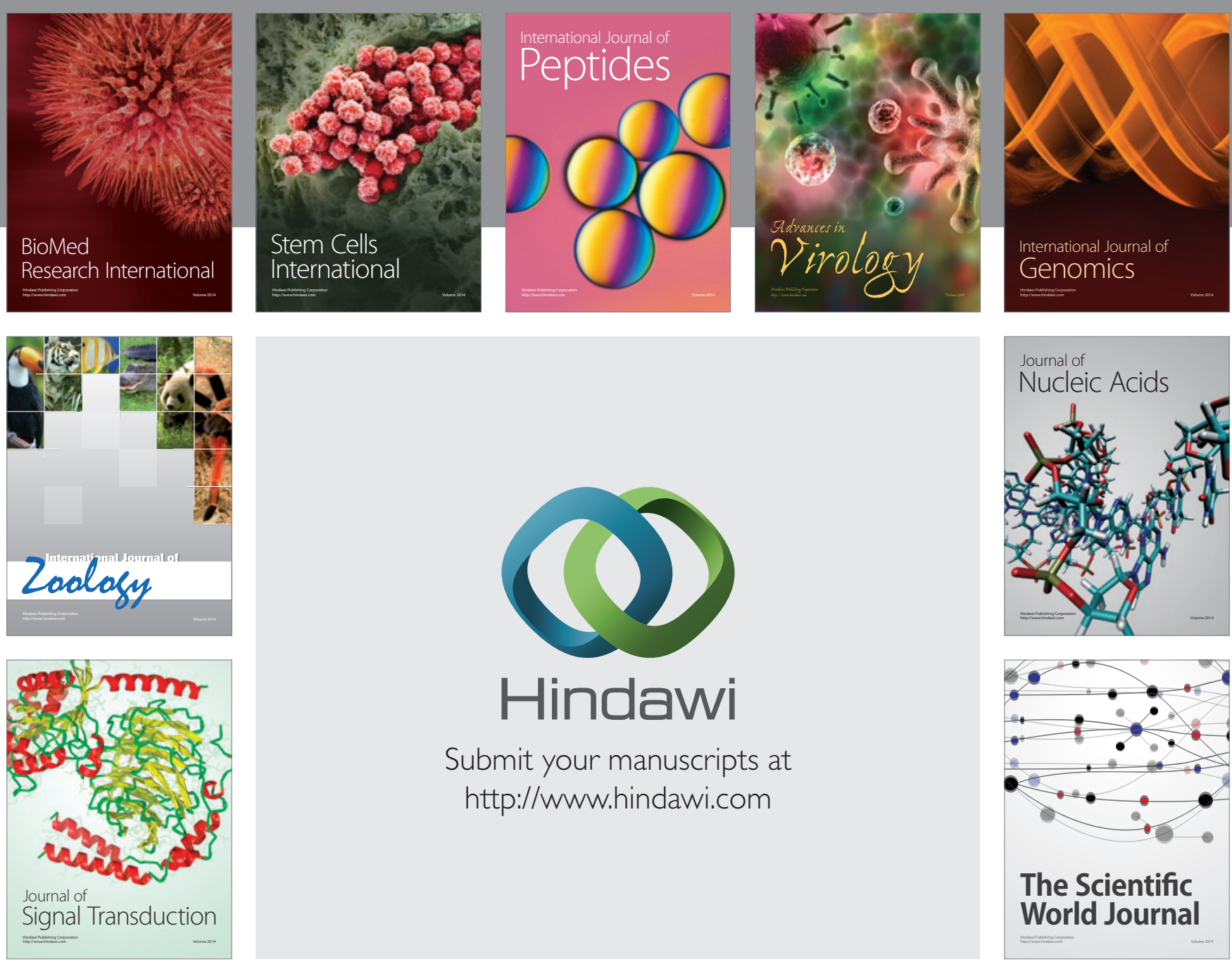

Submit your manuscripts at

http://www.hindawi.com
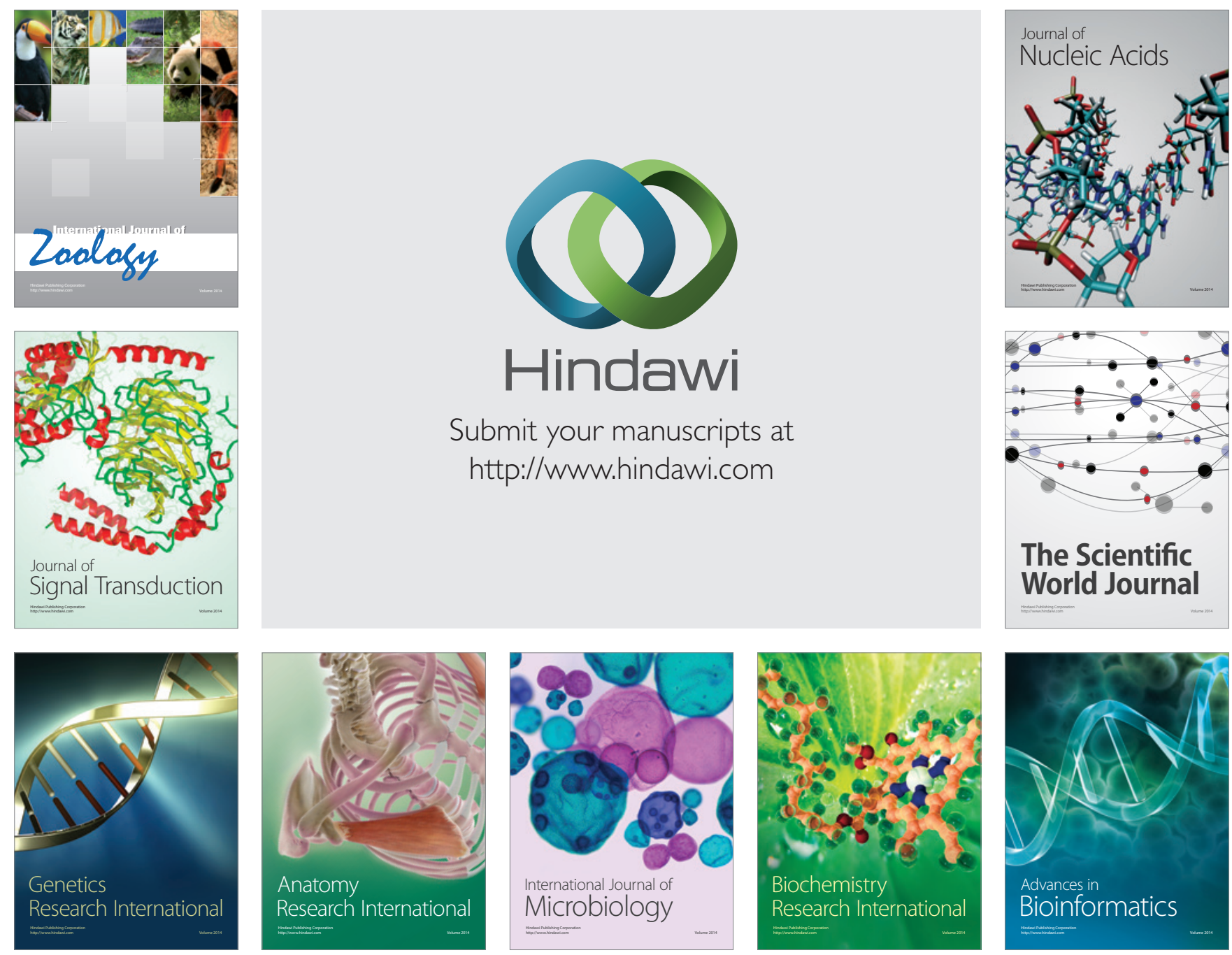

The Scientific World Journal
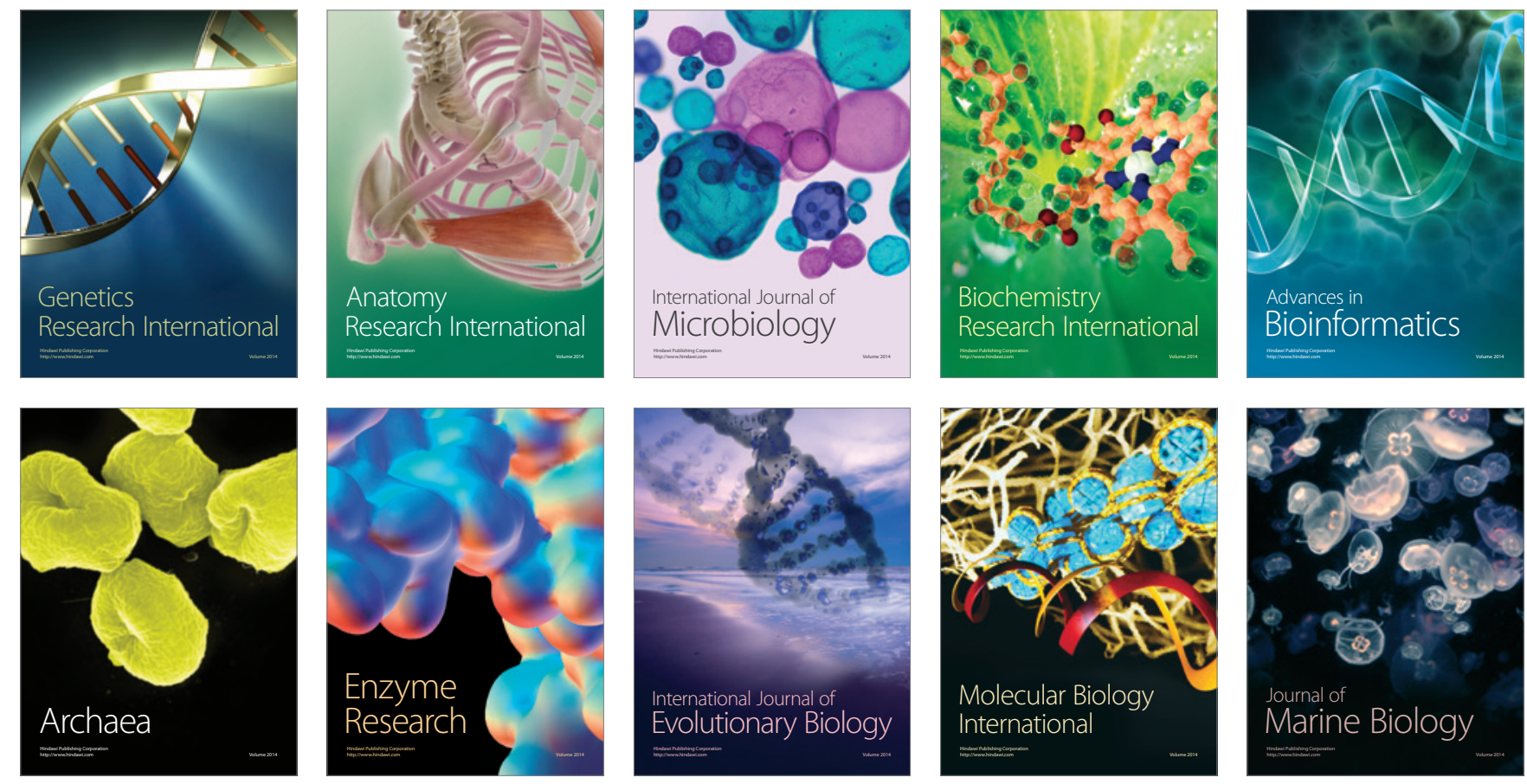